\title{
Editorial: Compulsory Interventions in Psychiatry: An Overview on the Current Situation and Recommendations for Prevention and Adequate Use
}

\author{
Christian G. Huber ${ }^{1 *}$ and Andres R. Schneeberger ${ }^{2,3,4}$ \\ ${ }^{1}$ Universitäre Psychiatrische Kliniken (UPK) Basel, Klinik für Erwachsene, University of Basel, Basel, Switzerland, ${ }^{2}$ Psychiatric \\ Services Grisons, Chur, Switzerland, ${ }^{3}$ Department of Psychiatry, Psychotherapy and Psychosomatics, Psychiatric Hospital, \\ University of Zurich, Zurich, Switzerland, ${ }^{4}$ Department of Psychiatry and Behavioral Sciences, Albert Einstein College of \\ Medicine, New York, NY, United States
}

Keywords: coercion, autonomy, involuntary treatment, seclusion, restraint, involuntary admission, community treatment order

\section{Editorial on the Research Topic}

Compulsory Interventions in Psychiatry: An Overview on the Current Situation and Recommendations for Prevention and Adequate Use

State-of-the-art clinical psychiatry seeks to provide successful treatment of persons with mental illness in a comprehensive approach integrating biological, psychological, social, and spiritual aspects (1). The focus on empowerment- and recovery-oriented strategies allows clinicians and people with mental illness to interact at the same level (2). However, illness symptoms sometimes prevent patients from fully understanding the potential benefits of treatment $(3,4)$. Aggression, violence, and self-endangering behavior in psychiatric patients are often used as justification for more restrictive policies in mental health care (4-7). Yet, current studies have shown that restrictive settings do not necessarily prevent self-harm and suicide $(8,9)$ and do not reduce all sorts of violence and aggression (10). Locked doors lead to a worse climate on the wards, directly affecting the therapeutic milieu and the treatment alliances $(11,12)$. In addition, patients who were mandated to psychiatric treatment might be more reluctant to receive treatment in the future [(3); Hachtel et al.]. While studies have shown that mandated treatments do also positively impact the outcome of illness, this is often mediated by an increase in services rendered rather than the direct effects of coercion (13).

Compulsory interventions aiming at patient and staff safety, as well as mandatory treatment may be necessary to ensure treatment for those who do not want to be treated (4). The goal is to protect mentally ill persons from self-harm, suicide, and detrimental consequences of untreated illness, and to protect relatives, healthcare professionals, and the general public from preventable aggression and violence (14). This gives rise to serious ethical problems and clinical challenges.

In the current Research Topic, Hoff elaborates on these ethical challenges. He discusses that coercive interventions have to be considered exceptional measures and may only be used under well-defined ethical and juridical conditions. Although guidelines should be adhered to, they cannot substitute individual case-based decisions. Furthermore, he recommends taking on the debate on autonomy in psychiatry, as facing this challenge might prove beneficial for psychiatry and its patients. Also focusing on patient autonomy, Scholten et al. discuss how different interpretations of psychiatric advance directives (PAD) may be useful for patients in future crises. They critically 
discuss interpretations of PAD promoting and undermining autonomy and propose that using supported decision-making and competence assessment may help to employ PAD successfully. As-like Hoff states-guidelines can only give general recommendations, Montaguti et al. discuss how clinical ethics consultation can be helpful to inform case-based decisions on coercive measures.

Following up to these ethical considerations, Oliva et al. discuss the legal preconditions for coercive measures. They explore if the judicial basis and the actual reasons for compulsory admission to psychiatric treatment in Turin (Italy) are mutually compatible and the authors critically discuss if changes in legislation are necessary. Becker and Forman bring up the point that psychiatric emergencies often occur in emergency rooms and outside of specific psychiatric settings. They discuss the role of implied consent in these emergency settings and explore the legal and ethical basis for acting in emergencies. Finally, Hachtel et al. discuss how the legal basis of mandated treatment influences psychiatric therapy and treatment outcome. They distinguish between the concepts of formal vs. perceived coercion and examine how psychiatry could fulfill a dual mandate of control and therapy and how it can be helpful even in the context of formal coercion. McMillan et al. also explore the issue of building a positive therapeutic alliance in the context of coercion. Using qualitative analysis of interviews with persons subjected to community treatment orders (CTO) and mental health professionals, they debate the role of trust and mistrust for the success and failure of reaching recovery in the frame of a CTO.

The legal framework regulating compulsory interventions has a direct impact on psychiatric practice. Radisic and Kolla focus on the situation in Ontario (Canada), where psychiatric inpatients who appeal to the Consent and Capacity Board (CCB) have the right to refuse medication until the $\mathrm{CCB}$ has come to a decision. They examine the frequency of seclusion and restraint in civil and forensic inpatients during this time and discuss how improving legislation and time to CCB decision could be beneficial for these patients. Mahler et al. take a look at the interplay of legal procedures and structural factors in their influence on the use of coercive measures in Germany. Arnold et al. examine the situation in Basel (Switzerland) and evaluate factors associated with compulsory admissions to psychiatric wards and with appeals against these admissions. Finally, Buadze et al. explore the position of defense lawyers in Switzerland on the availability of opioid agonist treatment vs. forced discontinuation in pre-trial detention and prisons. In these settings, availability of a specific treatment is partly controlled and moderated by custodians and legal professionals. Thus, there is a risk that this patient population does not receive interventions according to psychiatric state-of-the-art guidelines.

Addressing issues from social psychiatry and mental health services research, the original papers in the current Research Topic employ a broad set of qualitative and quantitative methods. Data for many of the publications in this Research Topic stem from clinical routine documentation. In this context, Fröhlich et al. examine the reliability of paper-based routine documentation in psychiatric inpatient care and find acceptable reliability, depending on the chosen documentation categories and variables. Together with the published literature, this indicates that both electronic and paper-based routine documentation can be used for health services research, but that their limitations have to be kept in mind.

Several publications focus on the frequency of coercive measures. In an effort to improve an overview on the use and effects of compulsory measures in psychiatry, de Bruijn et al. present a protocol for a systematic review on physical and pharmacological restraints. Saya et al. provide a narrative review on criteria, procedures, and future prospects of involuntary treatment in psychiatry around the world. Legal and ethical views and mental health services structures and traditions vary depending on country and sometimes region where patients are treated-and they have a considerable impact on practices regarding compulsory interventions. Thus, information on the situation in different countries provides important information and the basis for a discussion how the different settings should be developed.

Concerning the effects of coercive measures, Chieze et al. present a systematic review on the effects of seclusion and restraint in adult psychiatry. They conclude that, although the heterogeneity of the included studies limits interpretation, the overall results show negative effects of seclusion and restraint, and that more research is needed. Kersting et al. summarize the current literature on physical harm and death in the context of coercive measures in psychiatry in a systematic review, taking a look at a highly relevant but currently underresearched aspect of coercion in psychiatry.

As compulsory measures can often be avoided if a critical situation can be identified and successfully addressed early enough, research on the prediction of coercion is highly needed. To enhance our knowledge on the predictors of compulsory admission to treatment, Marty et al. study the characteristics of psychiatric emergency situations and the decision-making process leading to involuntary admission, and Lay et al. analyze the predictors of compulsory re-admission to psychiatric inpatient care. Günther et al. use machine learning to identify direct coercion in a high-risk subgroup of forensic patients with schizophrenia. And Hazewinkel et al. and Stepanow et al. explore the possibility of predicting seclusion by analyzing text entries from routine documentation in electronic medical records.

Several papers in the current Research Topic examine approaches to prevent or reduce compulsory interventions in psychiatry. Zinkler and Brophy et al. discuss the possibility to use supported decision making in the prevention of compulsory interventions and of community treatment orders in mental health care. Baumgardt et al. (a) study the effects of the introduction of the Safewards model on the use of coercive measures on two locked wards in Germany [please note that this paper has been updated according to the corrigendum in Baumgardt et al. (b)]. Schöttle et al. report that the introduction of an integrated care model in Hamburg (Germany) is connected with a reduction of involuntary admissions in patients with severe psychotic disorders. As Rabenschlag et al. discuss from a nursing perspective, de-escalation strategies can be useful for preventing and reducing coercion in psychiatry-including 
TABLE 1 | Overview on the papers published within the scope of the Research Topic.

\begin{tabular}{llll} 
Topic/Authors Title Article type \\
\hline
\end{tabular}

\section{Legal and ethical aspects}

Hoff

Scholten et al.

Montaguti et al.

Oliva et al.

Becker and Forman

Hachtel et al.

McMillan et al.

Radisic and Kolla

Arnold et al.

Mahler et al.

Buadze et al.

\section{Use of routine data}

Fröhlich et al.

\section{Frequency of coercive measures}

de Bruijn et al.

Saya et al.

\section{Effects of coercion}

Chieze et al.

Kersting et al.

\section{Prediction of coercion}

Marty et al.

Lay et al.

Günther et al.

Hazewinkel et al.

Stepanow et al.

\section{Prevention and reduction of coercion}

Zinkler

Brophy et al.

Baumgardt et al. (a)

Schöttle et al.

Rabenschlag et al.

Widmayer et al.
Compulsory interventions are challenging the identity of psychiatry

Psychiatric advance directives under the convention on the rights of persons with disabilities: why advance instructions should be able to override current preferences

Reflecting on the reasons pros and cons coercive measures for patients in psychiatric and somatic care: the role of clinical ethics consultation. A pilot study

Compulsory psychiatric admissions in an italian urban setting: are they actually compliant to the need for treatment criteria or arranged for dangerous not clinical condition?

Implied consent in treating psychiatric emergencies

Mandated treatment and its impact on therapeutic process and outcome factors Trust and community treatment orders

Right to appeal, non-treatment, and violence among forensic and civil inpatients awaiting incapacity appeal decisions in Ontario

Compulsory admission to psychiatric wards-who is admitted, and who appeals against admission?

Same, same but different: how the interplay of legal procedures and structural factors can influence the use of coercion

The accessibility of opioid agonist treatment and its forced discontinuation in swiss prisons-attitudes, perceptions and experiences of defense lawyers in dealing with detained persons using opioids

Reliability of paper-based routine documentation in psychiatric inpatient care and recommendations for further improvement

Physical and pharmacological restraints in hospital care: protocol for a systematic review

Criteria, procedures, and future prospects of involuntary treatment in psychiatry around the world: a narrative review

Effects of seclusion and restraint in adult psychiatry: a systematic review

Physical harm and death in the context of coercive measures in psychiatric patients: a systematic review

Characteristics of psychiatric emergency situations and the decision-making process leading to involuntary admission

Predictors of compulsory re-admission to psychiatric inpatient care

Identifying direct coercion in a high risk subgroup of offender patients with schizophrenia via machine learning algorithms

Text analysis of electronic medical records to predict seclusion in psychiatric wards: proof of concept

Narrative case notes have the potential to predict seclusion 3 days in advance: a mixed-method analysis

Supported decision making in the prevention of compulsory interventions in mental health care

Community treatment orders and supported decision-making

Preventing and reducing coercive measures - an evaluation of the implementation of the safewards model in two locked wards in Germany

Reduction of involuntary admissions in patients with severe psychotic disorders treated in the ACCESS integrated care model including therapeutic assertive community treatment

Nursing perspectives: reflecting history and informal coercion in de-escalation strategies

Could animal-assisted therapy help to reduce coercive treatment in psychiatry?
Perspective

Policy and Practice

Reviews

Original Research

Original Research

Opinion

Review

Original Research

Original Research

Original Research

Opinion

Original Research

Original Research

Clinical Study Protocol

Review

Systematic Review

Systematic Review

Original Research

Original Research

Original Research

Original Research

Original Research

Opinion

Original Research

Original Research

Original Research

Perspective

Mini Review 
TABLE 1 | Continued

\begin{tabular}{|c|c|c|}
\hline Topic/Authors & Title & Article type \\
\hline \multicolumn{3}{|l|}{ Staff and consumer perspectives } \\
\hline Sampogna et al. & $\begin{array}{l}\text { Perceived coercion among patients admitted in psychiatric wards: Italian results of } \\
\text { the EUNOMIA study }\end{array}$ & Original Research \\
\hline Efkemann et al. & $\begin{array}{l}\text { Ward atmosphere and patient satisfaction in psychiatric hospitals with different ward } \\
\text { settings and door policies. results from a mixed methods study }\end{array}$ & Original Research \\
\hline Fletcher, Hamilton et al. & $\begin{array}{l}\text { Safewards impact in inpatient mental health units in Victoria, Australia: staff } \\
\text { perspectives }\end{array}$ & Original Research \\
\hline Fletcher, Buchanan-Hagen et al. & $\begin{array}{l}\text { Consumer perspectives of safewards impact in acute inpatient mental health wards in } \\
\text { Victoria, Australia }\end{array}$ & Original Research \\
\hline Franke et al. & $\begin{array}{l}\text { Perceived institutional restraint is associated with psychological distress in forensic } \\
\text { psychiatric inpatients }\end{array}$ & Original Research \\
\hline Steinauer et al. & $\begin{array}{l}\text { Opening the doors of a substance use disorder ward-benefits and challenges from } \\
\text { a consumer perspective }\end{array}$ & Perspective \\
\hline Reisch et al. & $\begin{array}{l}\text { Comparing attitudes to containment measures of patients, health care professionals } \\
\text { and next of kin }\end{array}$ & Original Research \\
\hline Jaeger et al. & $\begin{array}{l}\text { Refusing medication therapy in involuntary inpatient treatment-a multiperspective } \\
\text { qualitative study }\end{array}$ & Original Research \\
\hline Soares and Pinto da Costa & $\begin{array}{l}\text { Experiences and perceptions of police officers concerning their interactions with } \\
\text { people with serious mental disorders for compulsory treatment }\end{array}$ & Original Research \\
\hline
\end{tabular}

informal coercion, which is otherwise often overlooked. The authors highlight the importance of the attitudes and values of the person perceiving aggression for their response to this behavior and advise that health care personnel should develop a critical awareness toward the use of coercive measures. Finally, Widmayer et al. pose the question if animal-assisted therapy could help to reduce coercive treatment in psychiatry and, based on positive findings in the current literature supporting this possibility, encourage future research on this topic.

Lastly, a number of papers assess staff and consumer perspectives on compulsory interventions in psychiatry. Sampogna et al. examine perceived coercion among inpatients of five psychiatric wards in Italy and its associations with treatment satisfaction. Efkemann et al. analyze ward atmosphere and patient satisfaction in locked, facultative locked and open door settings. Fletcher, Hamilton et al. explore the impact of the introduction of the Safewards model in inpatient mental health units in Victoria (Australia) on healthcare professionals and assess consumer perspectives in their second paper (Fletcher, Buchanan-Hagen et al.). Franke et al. address forensic psychiatric inpatient settings and examine perceived institutional restraint and psychological distress. Focusing specifically at substance use disorder wards, Steinauer et al. report healthcare personnel and consumer perspectives on the benefits and drawbacks of introducing an open door policy.

Concerning specific interventions and populations, Reisch et al. examine attitudes to containment measures of patients, next of kin, and health care professionals. Jaeger et al. conducted a qualitative study assessing the opinions of inpatients, their relatives, and healthcare professionals on not performing compulsory medication during involuntary inpatient treatment.
Lastly, Soares and Pinto da Costa examined the experiences and perceptions of police officers concerning their interactions with people with serious mental disorders for compulsory treatment.

Table 1 gives an overview on the papers published within the scope of the current Research Topic.

The primary focus of this Research Topic was to provide an overview on the current situation in clinical psychiatry and in psychiatric research, to collect scientific evidence on the prevention and adequate use of compulsory interventions, its effects and consequences. Now when finished, it also gives recommendations for mental health care professionals on the prevention of aggression and violence, the use of coercive measures and possible treatment alternatives to reduce forced interventions. In addition, it outlines future research strategies to advance the field and to ultimately approach the goal of optimal and safe treatment of this vulnerable population.

Of course, the number and distribution of submissions to a Research Topic cannot be considered representative for a research field. However, the considerable number of papers together with the current scientific literature show that research on compulsory interventions is a broad and active field in psychiatry. This mirrors a rising awareness of this issue in academic and clinical psychiatry. Ethical and legal aspects of coercion, the prediction, prevention and reduction of coercion, and consumer perspectives on coercion where the most prominent focus of submissions to this Research Topic. This shows that professionals within the field of psychiatry are critically evaluating in which situations and under which preconditions compulsory measures should be used and how they can be avoided. It acknowledges the importance of the field for healthcare professionals, patients, 
their relatives and the population, and shows that there is a willingness to strife for a minimal restrictive environment for the patients. From our view, this is a very positive development and fosters hope that we can successfully improve the situation for our patients and psychiatry now and in the future.

\section{REFERENCES}

1. Hochstrasser L, Frohlich D, Schneeberger AR, Borgwardt S, Lang UE, Stieglitz $\mathrm{RD}$, et al. Long-term reduction of seclusion and forced medication on a hospital-wide level: Implementation of an open-door policy over 6 years. Eur Psychiatry. (2018) 48:51-7. doi: 10.1016/j.eurpsy.2017.09.008

2. Huber CG, Sowislo JF, Schneeberger AR, Flury Bodenmann B, Lang UE. Empowerment - ein Weg zur Entstigmatisierung der psychisch Kranken und der Psychiatrie. Schweiz Arch Neurol Psychiatr. (2015) 166:22531. doi: $10.4414 /$ sanp.2015.00359

3. Fröhlich D, Schweinfurth N, Lang UE, Huber CG. Zwangsmassnahmen in der Psychiatrie. Lead Opin Neurol Psychiatr. (2017) 3:30-2.

4. Kowalinski E, Schneeberger AR, Lang UE, Huber CG. Safety through locked doors in psychiatry? In: Jakov G, Henking T, Nossek A, Vollmann J, editors. Beneficial Coercion in Psychiatry? Münster: Mentis (2017). p. 14762. doi: 10.30965/9783957438157_011

5. Schneeberger AR, Huber CG, Lang UE. Open wards in psychiatric clinics and compulsory psychiatric admissions. JAMA Psychiatry. (2016) 73:1293. doi: 10.1001/jamapsychiatry.2016.1738

6. Huber CG, Schottle D, Lambert M, Hottenrott B, Agorastos A, Naber D, et al. Brief Psychiatric Rating Scale - Excited Component (BPRS-EC) and neuropsychological dysfunction predict aggression, suicidality, and involuntary treatment in first-episode psychosis. Schizophr Res. (2012) 134:273-8. doi: 10.1016/j.schres.2011.1 2.002

7. Steinauer R, Huber CG, Petitjean S, Wiesbeck GA, Dursteler KM, Lang UE, et al. Effect of door-locking policy on inpatient treatment of substance use and dual disorders. Eur Addict Res. (2017) 23:87-96. doi: 10.1159/000458757

8. Huber CG, Schneeberger AR, Kowalinski E, Frohlich D, von Felten S, Walter $\mathrm{M}$, et al. Suicide risk and absconding in psychiatric hospitals with and without open door policies: a 15 year, observational study. Lancet Psychiatry. (2016) 3:842-9. doi: 10.1016/S2215-0366(16)30168-7

\section{AUTHOR CONTRIBUTIONS}

$\mathrm{CH}$ wrote the first draft of the manuscript. AS critically revised the manuscript and provided important intellectual contributions. All authors have read and approved the final version.

9. Huber CG, Borgwardt S, Lang UE, Schneeberger AR. Taking Off the blinders: the critical phase of suicidality doesn't end with discharge from inpatient treatment. Am J Bioethics. (2019) 19:93-4. doi: 10.1080/15265161.2019.1654023

10. Schneeberger AR, Kowalinski E, Frohlich D, Schroder K, von Felten S, Zinkler $\mathrm{M}$, et al. Aggression and violence in psychiatric hospitals with and without open door policies: a 15-year naturalistic observational study. J Psychiatr Res. (2017) 95:189-95. doi: 10.1016/j.jpsychires.2017.08.017

11. Blaesi S, Gairing SK, Walter M, Lang UE, Huber CG. Safety, therapeutic hold, and patient's cohesion on closed, recently opened, and open psychiatric wards. Psychiatr Prax. (2015) 42:76-81. doi: 10.1055/s-0033-1359871

12. Lo SB, Gaupp R, Huber C, Schneeberger A, Garic G, Voulgaris A, et al. Influence of an "open door policy" on ward climate: impact on treatment quality. Psychiatr Prax. (2018) 45:133-9. doi: 10.1055/s-0042-121784

13. Schneeberger AR, Huber CG, Lang UE, Muenzenmaier KH, Castille D, Jaeger $M$, et al. Effects of assisted outpatient treatment and health care services on psychotic symptoms. Soc Sci Med. (2017) 175:15260. doi: 10.1016/j.socscimed.2017.01.007

14. Lang UE, Borgwardt S, Walter M, Huber CG. Einführung einer "Offenen Tür Politik" - Was bedeutet diese konkret und wie wirkt sie sich auf Zwangsmaßnahmen aus? Recht Psychiatr. (2017) 35:72-9.

Conflict of Interest: The authors declare that the research was conducted in the absence of any commercial or financial relationships that could be construed as a potential conflict of interest.

Copyright (C) 2020 Huber and Schneeberger. This is an open-access article distributed under the terms of the Creative Commons Attribution License (CC BY). The use, distribution or reproduction in other forums is permitted, provided the original author(s) and the copyright owner(s) are credited and that the original publication in this journal is cited, in accordance with accepted academic practice. No use, distribution or reproduction is permitted which does not comply with these terms. 